\title{
Effects of endogenous ascorbic acid on resistance to high-temperature stress in excised rice leaves
}

\author{
Q.L. ZHANG, Y.X. WEI, and C.L. PENG ${ }^{+}$ \\ Guangzhou Key Laboratory of Subtropical Biodiversity and Biomonitoring, Guangdong Provincial Key Laboratory \\ of Biotechnology for Plant Development, College of Life Science, South China Normal University, \\ Guangzhou 510631, China
}

\begin{abstract}
Ascorbic acid (Asc) is a major plant antioxidant. L-galactono-1,4-lactone dehydrogenase (GLDH) is an enzyme that catalyzes the last step of Asc biosynthesis in higher plants. Effects of endogenous Asc on resistance to high-temperature stress were studied by using GLDH-overexpressed (GO-2) and GLDH-suppressed transgenic rice (GI-2) as experimental materials. After high-temperature treatment, the maximal quantum yield of PSII was significantly lower in GI-2, and higher in GO-2 compared to wild type rice. The content of reactive oxygen species (ROS) was the highest in GI-2. The higher Asc content resulted in lower lipid peroxidation in GO-2. The contents of chlorophyll, soluble proteins, and Rubisco large and small subunit were positively correlated to the Asc content. These results show that the higher Asc content reduced the accumulation of ROS and maintained the function of rice leaves. We suggest that the higher Asc content could improve the rice resistance to high-temperature stress.
\end{abstract}

Additional key words: ascorbic acid; high-temperature stress; reactive oxygen species; rice.

Rice is the staple crop for approximately half of the world population (Iizumi and Ramankutty 2016, Fairhurst and Dobermann 2002). In China, rice is mainly grown in summer. However, high temperatures in the summer cause damage to rice and affect the photosynthetic capacity (Bita and Gerats 2013) in rice-growing areas throughout the world (Kaneko et al. 2016). The high-temperature damage in rice mainly affects rice grain filling, which results in empty rice (Aquerreta et al. 2007). These criteria are followed to select the high-temperature-resistant rice varieties.

High temperature induces a series of physiological and biochemical reactions, therefore the attempt to improve the stability of physiological and biochemical in rice at high temperatures, such as the membrane system stability and the normal enzymatic reaction can significantly improve the plant photosynthetic performances (Xiong et al. 2017,
Jiang et al. 2003). Especially, after rice heading, when rice leaves enter the aging stage, the absorption and utilization of light energy are out of balance and excessive light could lead to a formation of ROS. The high temperature could affect the enzyme activities and physiological and biochemical reactions of ROS. Excessive ROS would destroy the photosynthetic apparatus (Hideg and Schreiber 2007), which would lead to a reduction in rice production.

Asc is an important antioxidant in plants (Akram et al. 2017, Potters et al. 2010). It directly scavenges the ROS, and serves as a coenzyme to scavenge $\mathrm{H}_{2} \mathrm{O}_{2}$ (Gest et al. 2013). The Asc is involved in physiological processes, such as antioxidation, senescence regulation, and stress response (Mekki et al. 2015, Chiang et al. 2017). The plants with insufficient Asc are sensitive to environmental factors. Under adverse conditions, Asc content is increased and is positively correlated with stress resistance in plants (Gallie 2013).

Received 5 October 2017, accepted 23 January 2018, published as online-first 21 June 2018.

${ }^{+}$Corresponding author; phone: +86-20-85217612; fax: +86-20-85215535; e-mail: pengchl@ ,scib.ac.cn

Abbreviations: Asc - ascorbic acid; Chl - chlorophyll; CK - normal temperature control group; DAB - diaminobenzidine; ETR electron transport rate; $F_{0}$ - minimal fluorescence yield of the dark-adapted state; $F_{m}$ - maximal fluorescence yield of the dark-adapted state; $F_{v}$ - variable fluorescence; $F_{v} / F_{m}$ - maximal quantum yield of PSII photochemistry; GI-2 - GLDH-suppressed transgenic rice; GLDH - 1-galactono-1,4-lactone dehydrogenase; GO-2 - GLDH-overexpressed transgenic rice; HT - high-temperature treatment group; MDA - malondialdehyde; NBT - nitrotetrazolium blue; RL - Rubisco large subunit; RS - Rubisco small subunit; RH - relative humidity; ROS - reactive oxygen species; TCA - trichloroacetic acid; ZH-11 - wild-type.

Acknowledgements: The authors are very grateful to Professor Xingxiang Peng (South China Agricultural University, China) for the generous gift of rice seeds. This work was supported by National Natural Science Foundation of China $(31570398,31270287)$, the key project of Guangdong Province Natural Science Foundation 2015A030311023. 
It has been reported that 1-galactono-1,4-lactone dehydrogenase (GLDH) was the rate-limiting enzyme in Asc synthesis (Smirnoff et al. 2001), and the Asc content in plants was positively correlated with the expression of GLDH gene (Landi et al. 2015, Zhang et al. 2016, Tokunaga et al. 2005). GLDH up-regulated and the GLDH down-regulated rice strains had been successfully constructed (Yu et al. 2010, 2017).

In this study, we investigated the effects of endogenous Asc on resistance to high-temperature stress in excised rice leaves. The consequences of a changes in Asc to lipid peroxidation, Rubisco protein, soluble protein, ROS, chlorophyll $(\mathrm{Chl})$, and $\mathrm{Chl}$ fluorescence parameters were compared in GO-2, wild type (ZH-11), and GI-2. Our results suggested that the higher Asc content could improve the resistance capacity of rice to high-temperature stress.

Oryza sativa L. cv. Zhonghua 11 (ZH-11), a previously described homozygous GLDH-overexpressing transgenic rice GO-2, and the GLDH-suppressed transgenic rice GI-2 (Liu et al. 2011) were used in this study. The pasteurized seeds were planted in an artificial climate incubator and grown under controlled conditions with an average temperature of $28^{\circ} \mathrm{C}$ dark $/ 33^{\circ} \mathrm{C}$ light, $80 \%$ humidity, and a photoperiod of $10 \mathrm{~h}$ dark $/ 14 \mathrm{~h}$ light [100 $\mu$ mol(photon) $\left.\mathrm{m}^{-2} \mathrm{~s}^{-1} \mathrm{PAR}\right]$. They were transplanted to the field after $14 \mathrm{~d}$. When the plants began to tassel, the rice cultivars with consistent growth were selected and used for the experiments.

The flag leaves were cut at the flowering stage, wrapped with wet gauze, and placed in a water bath at $43^{\circ} \mathrm{C}$ for $1 \mathrm{~h}$ as the high-temperature treated group (HT). The flag leaves wrapped with wet gauze placed at $25^{\circ} \mathrm{C}$ for $1 \mathrm{~h}$ were considered as the control group (CK).

Total RNA was extracted from flag leaves using an Omega R6827 kit (Takara, Japan) in accordance with the manufacturer's instructions. First-strand cDNA was synthesized with oligo (dT) primers using a $M-M L V$ reverse transcriptase kit (Takara, Japan), and then the cDNAs were stored at $-80^{\circ} \mathrm{C}$ until further analysis. Quantitative real-time PCR (q-PCR) was performed according to the manufacturer's instructions. The q-PCR primer sequences were as follows: $G L D H-\mathrm{F}$ (5'-CGGCGGCATCATTCAGGTT-3') and GLDH-R (5'-AAGCCCACAGCGAGCAAGATA-3'). The primer sequences of reference genes were as follows: Os $U B Q-\mathrm{F}$ (5'-CCAGGACAAGATGATCTGCC-3') and $O s U B Q-\mathrm{R}$ (5'-AAGAAGCTGAAGCATCCAGC-3'). Reactions were performed using SYBR ${ }^{\circledR}$ Premix EX Tap ${ }^{\mathrm{TM}}$ II (Takara, Japan) in a real-time PCR device (7500 Sequence Detection system, Applied Biosystems, Japan). The expression levels of the mRNA of $G L D H$ genes were normalized according to the reference gene $O s U B Q$. Normalization and fold changes were calculated using the $\Delta \Delta^{\mathrm{Ct}}$ method of Livak and Schmittgen (2001).

Fresh flag leaves of $0.1 \mathrm{~g}$ were cut and fully ground and homogenized in $1 \mathrm{~mL}$ of $6 \%$ trichloroacetic acid (TCA) solution in an ice bath. The homogenate was centrifuged at $13,000 \times g$ at $4^{\circ} \mathrm{C}$ for $10 \mathrm{~min}$. The supernatant was used for the analysis of Asc contents according to Kampfenkel et al. (1995).

Rubisco protein determination was carried out according to Liu et al. (2011). Fresh leaves of $0.1 \mathrm{~g}$ were homogenized in $2 \mathrm{~mL}$ of grinding medium and the homogenate was centrifuged at $13,000 \times g$ for $10 \mathrm{~min}$. Supernatant $(50 \mu \mathrm{L})$ was added to an equal volume of sample buffer and incubated in boiling water for $5 \mathrm{~min}$. The samples $(10 \mu \mathrm{L})$ were loaded onto gels containing $12.5 \%(\mathrm{w} / \mathrm{v})$ resolving polyacrylamide gel with a $4 \%(\mathrm{w} / \mathrm{v})$ polyacrylamide gel stacker. After gel was stained with Coomassie Brilliant Blue R-250 (Sigma, USA) and destained overnight with gentle shaking to make sure that its background turned colorless. The molecular mass of the large and small subunit of Rubisco are 55 and $15 \mathrm{kDa}$, respectively. The SDS-PAGE gel was scanned using the GelDoc-It Imaging System (UVP, Upland, CA, USA) and relative grey value of each band detected by using TotalLab Quant software (TotalLab, Newcastle upon Tyne, UK). On the other hand, the supernatant was collected and diluted for 50 times. Then $250 \mu \mathrm{L}$ of diluted supernatant was added into the same volume of Bradford solution. After $5 \mathrm{~min}$, and the absorbance was read at a wavelength of $595 \mathrm{~nm}$ (UV-Vis 2450 spectrophotometer, Shimadzu, Tokyo, Japan).

Fresh leaves of $0.1 \mathrm{~g}$ were extracted with $10 \mathrm{~mL}$ of $80 \%$ $(\mathrm{v} / \mathrm{v})$ acetone. The absorption of the extracts was measured at 663 and $645 \mathrm{~nm}(U V$-Vis 2450, Shimadzu, Tokyo, Japan). The $80 \%$ (v/v) acetone was used as a blank. Chl $a$ and Chl $b$ were determined according to the method of Lichtenthaler (1987).

After HT, the leaves were dipped quickly into potassium phosphate buffer $(50 \mathrm{mM}, \mathrm{pH} 7.0)$ containing $1 \mathrm{mg}$ (diaminobenzidine, DAB) $\mathrm{mL}^{-1}$ and potassium phosphate buffer ( $50 \mathrm{mM}, \mathrm{pH} 6.4$ ) containing $1 \mathrm{mg}$ (nitrotetrazolium blue, NBT) $\mathrm{mL}^{-1}$, respectively. Then they were subjected to rapid vacuum suction for $20 \mathrm{~min}$. The leaves were immediately put in the dark for $8 \mathrm{~h}$ and $12 \mathrm{~h}$ (stained by $\mathrm{DAB}$ and NBT). The leaves were transferred into a boiling methanol solution $(75 \%, \mathrm{v} / \mathrm{v})$ to bleach and remove Chl. Finally, the leaves were photographed to detect the presence of $\mathrm{H}_{2} \mathrm{O}_{2}$ and $\mathrm{O}_{2}{ }^{\cdot-}$ (Liu et al. 2007).

The leaves from the rice plants were cut, wrapped with wet gauze, and placed in the dark for dark adaptation for $30 \mathrm{~min}$. After dark adaptation, a portable fluorescence analyzer (PAM-2100, Walz, Effeltrich, Germany) was used to measure the minimum fluorescence, $\mathrm{F}_{0}$, and the maximum fluorescence, $F_{m}$, under a $6,000 \mu$ mol(photon) $\mathrm{m}^{-2} \mathrm{~s}^{-1}$ of saturating pulse. The actinic light used in the measurement of the parameters was $150 \mu \mathrm{mol}$ (photon) $\mathrm{m}^{-2} \mathrm{~s}^{-1}$. The maximum efficiency of PSII $\left(\mathrm{F}_{\mathrm{v}} / \mathrm{F}_{\mathrm{m}}\right.$, calculated as $\left.1-\mathrm{F}_{0} / \mathrm{F}_{\mathrm{m}}\right)$, effective quantum yield of PSII photochemistry $\left(\Phi_{\mathrm{PSII}}\right.$, calculated as Yield $\left.=\Phi_{\mathrm{PSII}}=1-\mathrm{F}_{\mathrm{s}} / \mathrm{F}_{\mathrm{m}}{ }^{\prime}\right)$, and the electron transport rate $(\mathrm{ETR}$, calculated as ETR $=$ Yield $\times$ PAR $\times 0.5 \times 0.84)($ Oxborough and Baker 1997) .

Lipid peroxidation was evaluated by measuring the 
malondialdehyde (MDA) content. Flag leaves $(0.2 \mathrm{~g})$ were homogenized in $2 \mathrm{~mL}$ of grinding medium and the homogenate was centrifuged at $4,390 \times g$ for $10 \mathrm{~min}$. Supernatant $(2 \mathrm{~mL})$ was added to an equal volume of $0.67 \%$ 2-thiobarbituric acid, and the solution was kept in a boiling water bath for about $15 \mathrm{~min}$ and centrifuged at $4,390 \times g$ for $10 \mathrm{~min}$. The supernatant was evaluated for light absorption at 532, 600, and $450 \mathrm{~nm}$ (UV-Vis 2450, Shimadzu, Tokyo, Japan). The MDA amount was calculated according to the method of Heath and Packer (1968).

All experiments were conducted in triplicate. Values were expressed as means $\pm \mathrm{SD}$. The results were subjected to a one-way analysis of variance (ANOVA) with Duncan's multiple range test between means at $p<0.05$. Data were processed by using the SigmaPlot software (version 12.0, SYSTAT Software Inc., Richmond, CA, USA).

Significant differences were observed in the expression of $G L D H$ gene in leaves of three rice cultivars (Fig. 1A). $G L D H$ expression was 30-times higher in GO-2 plants than that in $\mathrm{ZH}-11$, while the expression of $G L D H$ in GI-2 plants was 6-times lower. Similarly, there were significant differences in the Asc content of the GO-2, ZH-11, and GI-2 leaves. The content of Asc in GO-2 was 3.1- and 10-fold higher than those of the ZH-11 and GI-2, respectively (Fig. $1 B$ ).

In plants, the $\mathrm{O}_{2}{ }^{-}$and $\mathrm{H}_{2} \mathrm{O}_{2}$ react with NBT and DAB to generate blue and tan insoluble precipitations, respectively, on the blade surface. The leaves of the plants subjected to HT were stained with NBT and DAB. The results showed that the leaves of GO-2 strain exhibited the lightest color, while the leaves of $\mathrm{ZH}-11$ and GI-2 showed deep staining (Fig. 1C,D). Using NBT and DAB histochemical staining, we detected the corresponding accumulation of greater amounts of $\mathrm{O}_{2}{ }^{-}$and $\mathrm{H}_{2} \mathrm{O}_{2}$, respectively, in the flag leaves of GI-2 plants.

Polyacrylamide gel electrophoresis did not show the obvious differences in the content of RL and RS between the leaves of GI-2, GO-2, and $\mathrm{ZH}-11$ under normal temperature conditions. However, the HT decreased the content of RL and RS in rice leaves. The content of RL decreased by 34,32 , and $41 \%$ in the leaves of GO-2, $\mathrm{ZH}$ 11 , and GI-2, respectively (Fig. $1 E$ ). The content of RS decreased in 31,33 , and $70 \%$ in the leaves of GO-2, $\mathrm{ZH}-$ 11 , and GI-2, respectively (Fig. $1 F$ ). The polyacrylamide gel electrophoresis showed that high temperature resulted in a significant decrease in the content of RL and RS. The content of RS was the lowest one after HT (Fig. 1G).

The content of MDA reflects the lipid peroxidation. The HT increased the membrane lipid peroxidation in rice leaves (Fig. 2A). The highest lipid peroxidation was observed in the GI-2 plants. The MDA content increased by 35,63 , and $168 \%$ in GO-2, ZH-11, and GI-2, respectively. The soluble protein content in rice leaves decreased after HT, with the largest decrease in the GI-2 plants (29\%). The soluble protein content of GO-2 and ZH-11 decreased by 18 and $22 \%$, respectively (Fig. $2 B$ ).
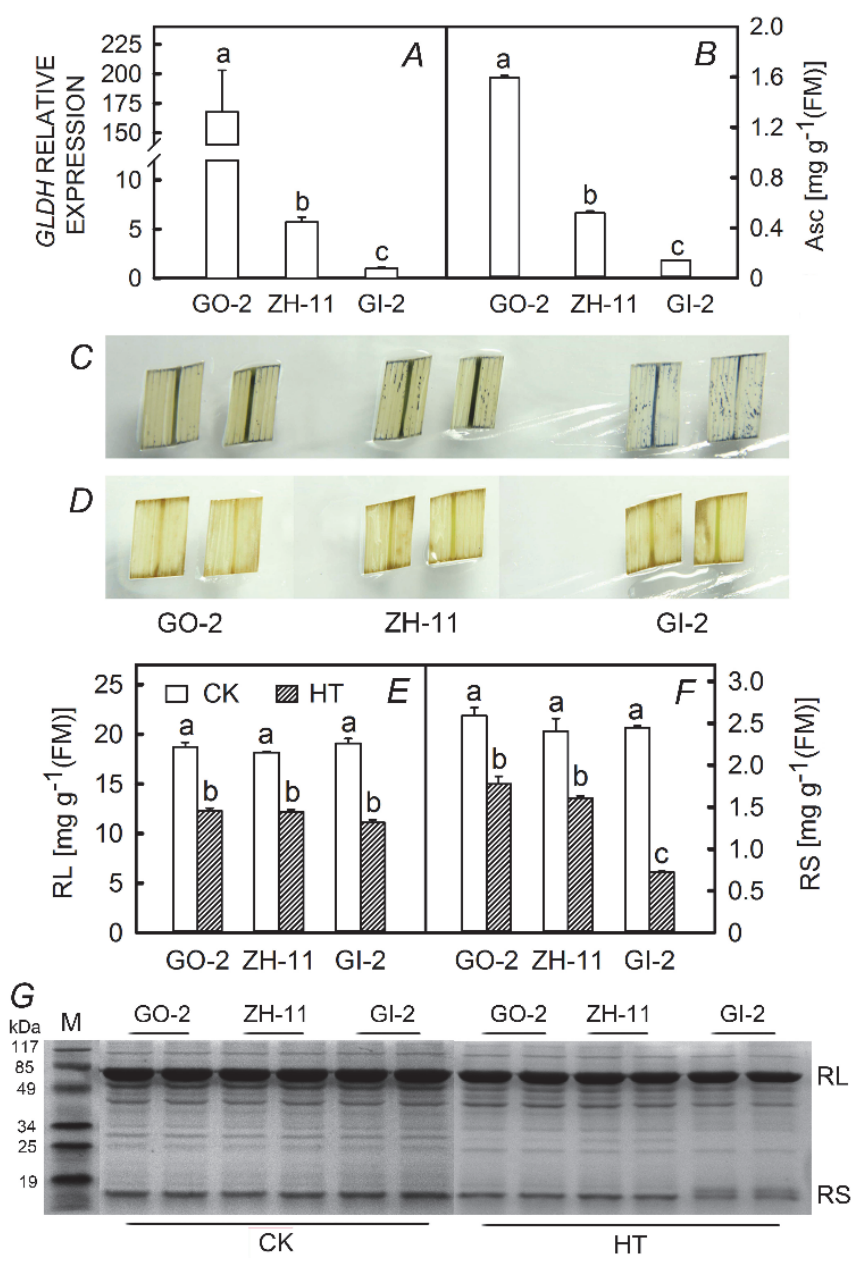

Fig. 1. Relative expression of 1-galactono-1,4-lactone dehydrogenase gene $(\mathrm{GLDH} ; A)$ and ascorbic acid content (Asc; $B)$ in flag leaves of GO-2, ZH-11, and GI-2 plants. Nitroblue tetrazolium staining $(\mathrm{NBT} ; C)$ and diaminobenzidine staining $(\mathrm{DAB} ; D)$ showed the accumulation of superoxide anion $\left(\mathrm{O}_{2}{ }^{-}\right)$ and hydrogen peroxide $\left(\mathrm{H}_{2} \mathrm{O}_{2}\right)$ in the flag leaves of GO-2, ZH-11, and GI-2 plants after high-temperature treatment. The changes of Rubisco large subunit (RL; $E$ ) and Rubisco small subunit (RS; $F$ ) in flag leaves of GO-2, ZH-11, and GI-2 plants after high-temperature treatment $(\mathrm{CK}$, normal temperature control group; HT, high-temperature treatment group). $(G)$ Detection of the changes in RL and RS by SDS-PAGE in flag leaves of GO-2, ZH-11, and GI-2 plants after high-temperature treatment. Values are means $\pm \operatorname{SD}(n=5)$. Different letters above bars indicate statistical significance $(p<0.05)$.

Insignificant differences in yield, ETR, and $F_{v} / F_{m}$ were found in the leaves of the three genotypes of rice at normal temperature (Fig. $2 C, D, E$ ). However, the HT significantly decreased the $\mathrm{Chl}$ fluorescence parameters in GO-2, $\mathrm{ZH}-11$, and GI-2. The decline in yield and $\mathrm{F}_{\mathrm{v}} / \mathrm{F}_{\mathrm{m}}$ of GO-2 plants were the smallest ( 30 and $46 \%$, respectively), while the maximum decline were observed in GI-2 plants (81 and $80 \%$, respectively) (Fig. 2C,E). The total Chl content of the leaves of the three genotypes decreased after HT compared with those under normal temperature. The 


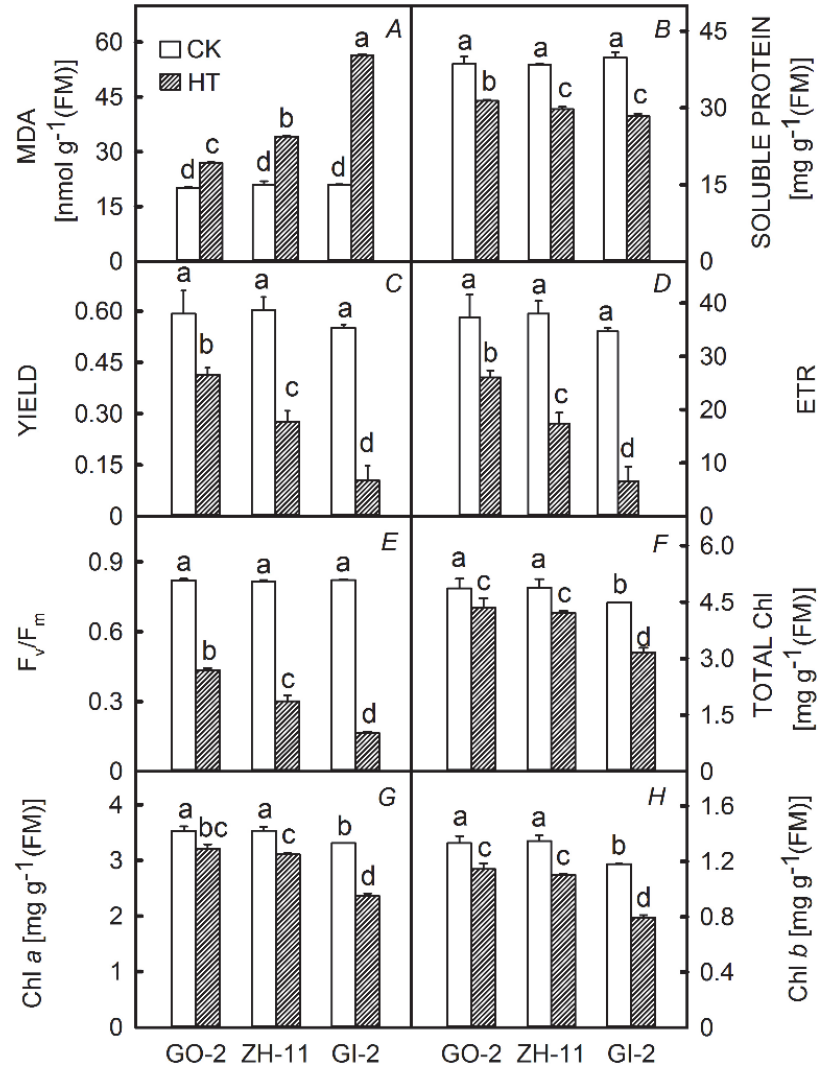

Fig. 2. Changes of malondialdehyde (MDA; $A$ ), soluble protein $(B)$, yield $(C)$, and electron transport rate $(\mathrm{ETR} ; D)$ in flag leaves of GO-2, ZH-11, and GI-2 plants after high-temperature treatment. Changes in maximal quantum yield of PSII photochemistry $\left(\mathrm{F}_{\mathrm{v}} / \mathrm{F}_{\mathrm{m}} ; E\right)$, total chlorophyll $(\mathrm{Chl})$ contents $(F)$, Chl $a$ contents $(G)$, and $\mathrm{Chl} b$ contents $(H)$ in flag leaves of GO-2, ZH-11, and GI-2 plants after high-temperature treatment. CK, normal temperature control group; HT, high-temperature treatment group. Values are means $\pm \mathrm{SD}(n=5)$. Different letters above bars indicate statistical significance $(p<0.05)$.

maximum decrease of total Chl content in GI-2 leaves was $30 \%$. The GO-2 and ZH-11 plants showed a reduction of only $10 \%$ (Fig. $2 F$ ). Moreover, the change trends of Chl $a$ content and $\mathrm{Chl} b$ content in the leaves of the three genotypes were consistent with the total $\mathrm{Chl}$ content (Fig. 2G,H).

Plants are exposed to a variety of adverse environmental stresses during growth, and HT stress is one of them. When plants were stressed, they produced higher amounts of ROS. If ROS cannot be removed in time, they would damage the plant (Tanaka and Hadwiger 2017, Hemavathi et al. 2009). There were two major systems for the removal of ROS in plants: the enzymatic system and the nonenzymatic system; in both, Asc plays an important

\section{References}

Akram N.A., Shafiq F., Ashraf M.: Ascorbic acid-A potential oxidant scavenger and its role in plant development and abiotic role (Foyer et al. 1994, Asada 2000). It has been reported that endogenous Asc could effectively scavenge ROS in plants and increase the resistance of rice leaves and stress tolerance of Arabidopsis thaliana (Huang and Osborne 2002). In this study, changes in the Asc content and tissue localization of ROS showed that the accumulation of ROS in rice leaves was closely related to the content of Asc. Thus, Asc could effectively reduce the accumulation of ROS.

ROS, as highly toxic substances, can constantly attack the biological macromolecules in plants, degrade biological macromolecules, and destroy the integrity of membranes (Dhindsa et al. 1982, Barna et al. 2012, Taheri et al. 2014). MDA is an important indicator of lipid peroxidation. The integrity of membrane lipids is closely related to the MDA content (Heath and Packer 1968). Our previous studies showed that Asc is beneficial for maintaining membrane stability during the induction of senescence in rice leaves ( $\mathrm{Yu}$ et al. 2017). Our results showed that MDA in the leaves of GI-2 plants increased by $168 \%$ after HT (Fig. 2A), indicating that the integrity of the membrane lipids of the leaves in the HT environment was seriously damaged. The soluble protein content was more reduced in GI-2 plants than that in GO-2 plants. These results indicate that Asc could improve the HT resistance of rice.

Rubisco is an important protein in the dark reactions of photosynthesis, and its integrity and content determine the formation of photosynthetic organic compounds (Perdomo et al. 2017, Bi et al. 2017). Chl is important for light use in photosynthesis, and its integrity and content determine the efficiency of light reactions (Liang et al. 2017). Rubisco protein and Chl content were positively correlated with the Asc content. Asc had protective effects on photosynthetic organs and increased biomass by minimizing the loss of photosynthestic performance (Yabuta et al. 2002, $\mathrm{Yu}$ et al. 2010). The results showed that the Rubisco protein and $\mathrm{Chl}$ content of the leaves of three different plants decreased after treatment at HT, while the increase in Asc reduced the degradation of Rubisco protein and Chl. $\mathrm{F}_{\mathrm{v}} / \mathrm{F}_{\mathrm{m}}$ represents the maximum efficiency of PSII. $\mathrm{F}_{\mathrm{v}} / \mathrm{F}_{\mathrm{m}}$ is one of the most commonly used parameters, and its reduction could reflect the effects of stress on the plant photosynthesis (Maxwell and Johnson 2000, Jägerbrand and Kudo 2016). Our results showed that $F_{v} / F_{m}$ of the rice leaves was significantly reduced after HT. Moreover, the decrease of $\mathrm{F}_{\mathrm{v}} / \mathrm{F}_{\mathrm{m}}$ (GO-2: $46 \%$, GI-2: 80\%) in leaves of three different rice genotypes showed that Asc effectively maintained the stability of photosynthetic functions. The above results showed that the increase of endogenous Asc could reduce the accumulation of ROS in rice leaves under high-temperature stress, reduce the toxic effect of ROS, and maintain the stability of rice leaves function.

stress tolerance. - Front. Plant Sci. 8: 613, 2017.

Aquerreta J., Iguaz A., Arroqui C. et al.: Effect of high 
temperature intermittent drying and tempering on rough rice quality. - J. Food Eng. 80: 611-618, 2007.

Asada K.: The water-water cycle as alternative photon and electron sinks. - Philos. T. Roy. Soc. Lond. 355: 1419-1431, 2000.

Barna B., Fodor J., Harrach B.D. et al.: The Janus face of reactive oxygen species in resistance and susceptibility of plants to necrotrophic and biotrophic pathogens. - Plant Physiol. Bioch. 59: 37-43, 2012.

Bi H., Liu P., Jiang Z. et al.: Overexpression of the rubisco activase gene improves growth and low temperature and weak light tolerance in Cucumis sativus L. - Physiol. Plantarum 161: 224-234, 2017.

Bita C.E., Gerats T.: Plant tolerance to high temperature in a changing environment: scientific fundamentals and production of heat stress-tolerant crops. - Front. Plant Sci. 4: 273, 2013.

Chiang C.M., Chen C.C., Chen S.P. et al.: Overexpression of the ascorbate peroxidase gene from eggplant and sponge gourd enhances flood tolerance in transgenic Arabidopsis. - J. Plant Res. 130: 373-386, 2017.

Dhindsa R.S., Plumb-Dhindsa P.L., Reid D.M.: Leaf senescence and lipid peroxidation: Effects of some phytohormones, and scavengers of free radicals and singlet oxygen. - Physiol. Plantarum 56: 453-457, 1982.

Fairhurst T.H., Dobermann A.: Rice in the global food supply. Better Crops Internat. 16: 3-6, 2002.

Foyer C.H., Lelandais M., Kunert K.J.: Photooxidative stress in plants. - Physiol. Plantarum 92: 696-717, 1994.

Gallie D.R.: The role of 1-ascorbic acid recycling in responding to environmental stress and in promoting plant growth. - J. Exp. Bot. 64: 433-443, 2013.

Gest N., Gautier H., Stevens R.: Ascorbate as seen through plant evolution: the rise of a successful molecule. - J. Exp. Bot. 64: 33-53, 2013.

Heath R.L., Packer L.: Photoperoxidation in isolated chloroplasts: I. Kinetics and stoichiometry of fatty acid peroxidation. - Arch. Biochem. Biophys. 125: 189-198, 1968.

Hemavathi, Chandramaprakash U., Koeun Y. et al.: Overexpression of strawberry D-galacturonic acid reductase in potato leads to accumulation of vitamin $\mathrm{C}$ with enhanced abiotic stress tolerance. - Plant Sci. 177: 659-667, 2009.

Hideg E., Schreiber U.: Parallel assessment of ROS formation and photosynthesis in leaves by fluorescence imaging. Photosynth. Res. 92: 103-108, 2007.

Huang Z., Osborne D.J.: Endo-glycanhydrolases activities in Artemisia sphaerocephala (Asteraceae) mucilaginous achene germination process. - Acta Bot. Sinica 44: 753-756, 2002.

Iizumi T., Ramankutty N.: Changes in yield variability of major crops for 1981-2010 explained by climate change. - Environ. Res. Lett. 11: 34003, 2016.

Jägerbrand A.K., Kudo G.: Short-term responses in maximum quantum yield of PSII $\left(\mathrm{F}_{\mathrm{v}} / \mathrm{F}_{\mathrm{m}}\right)$ to ex situ temperature treatment of populations of bryophytes originating from different sites in Hokkaido, Northern Japan. - Plants 5: E22, 2016.

Jiang H., Dian W., Wu P.: Effect of high temperature on fine structure of amylopectin in rice endosperm by reducing the activity of the starch branching enzyme. - Phytochemistry 63: 53-59, 2003.

Kampfenkel K., van Motagu M., Inzé D.: Extraction and determination of ascorbate and dehydroascorbate from plant tissue. Anal. Biochem. 225: 165-167, 1995.

Kaneko K., Sasaki M., Kuribayashi N. et al.: Proteomic and glycomic characterization of rice chalky grains produced under moderate and high-temperature conditions in field system. -
Rice 9: 1-16, 2016

Landi M., Fambrini M., Basile A. et al.: Overexpression of $L$ galactono-1,4-lactone dehydrogenase, (L-GalLDH) gene correlates with increased ascorbate concentration and reduced browning in leaves of Lactuca sativa, L. after cutting. - Plant Cell Tiss. Org. 123: 109-120, 2015.

Liang Y., Urano D., Liao K.L. et al.: A nondestructive method to estimate the chlorophyll content of Arabidopsis seedlings. Plant Methods 13: 26, 2017.

Lichtenthaler H.K.: Chlorophylls and carotenoids: Pigments of photosynthetic biomembranes. - Method Enzymol. 148: 350382, 1987.

Liu Y., Ren D., Pike S. et al.: Chloroplast-generated reactive oxygen species are involved in hypersensitive response-like cell death mediated by a mitogen-activated protein kinase cascade. - Plant J. 51: 941-954, 2007.

Liu Y., Yu L., Wang R.: Level of ascorbic acid in transgenic rice for 1-galactono-1,4-lactone dehydrogenase overexpressing or suppressed is associated with plant growth and seed set. - Acta Physiol. Plant. 33: 1353-1363, 2011.

Livak K.J., Schmittgen T.D.: Analysis of relative gene expression data using real-time quantitative PCR and the 2(-Delta Delta C(T)) Method. - Methods 25: 402-408, 2001.

Maxwell K., Johnson G.N.: Chlorophyll fluorescence - a practi cal guide. - J. Exp. Bot. 51: 659-668, 2000.

Mekki E.D., Hussien H.A., Salem H.: Role of glutathione, ascorbic acid and $\alpha$-tocopherol in alleviation of drought stress in cotton plants. - Int. J. Chemtech. Res. 8: 1573-1581, 2015.

Oxborough K., Baker N.R.: Resolving chlorophyll a fluorescence images of photosynthetic efficiency into photochemical and non-photochemical components - calculation of $\mathrm{qP}$ and $\mathrm{F}_{\mathrm{v}}{ }^{\prime} / \mathrm{F}_{\mathrm{m}}{ }^{\prime}$ without measuring $\mathrm{F}_{0}{ }^{\prime}$. - Photosynth. Res. 54:135-142, 1997.

Perdomo J.A., Capó-Bauçà S., Carmo-Silva E. et al.: Rubisco and rubisco activase play an important role in the biochemical limitations of photosynthesis in rice, wheat, and maize under high temperature and water deficit. - Front. Plant Sci. 8: 490, 2017.

Potters G., Horemans N., Jansen M.A.K.: The cellular redox state in plant stress biology - a charging concept. - Plant Physiol. Bioch. 48: 292-300, 2010.

Smirnoff N., Conklin P.L., Loewus F.A.: Biosynthesis of ascorbic acid in plants: a renaissance. - Annu. Rev. Plant Phys. 52: 437-467, 2001.

Taheri P., Irannejad A., Goldani M. et al.: Oxidative burst and enzymatic antioxidant systems in rice plants during interaction with Alternaria alternate. - Eur. J. Plant Pathol. 140: 829-839, 2014.

Tanaka K., Hadwiger L.A.: Nonhost resistance: Reactive oxygen species (ROS) signal causes DNA damage prior to the induction of PR genes and disease resistance in pea tissue. Physiol. Mol. Plant P. 98: 18-24, 2017.

Tokunaga T., Miyahara K., Tabata K. et al.: Generation and properties of ascorbic acid-overproducing transgenic tobacco cells expressing sense RNA for L-galactono-1,4-lactone dehydrogenase. - Planta 27: 854-863, 2005.

Xiong D., Ling X., Huang J. et al.: Meta-analysis and doseresponse analysis of high temperature effects on rice yield and quality. - Environ Exp Bot. 141: 1-9, 2017.

Yabuta Y., Motoki T., Yoshimura K. et al.: Thylakoid membrane-bound ascorbate peroxidase is a limiting factor of antioxidative systems under photo-oxidative stress. - Plant $\mathrm{J}$. 32: 915-925, 2002.

Yu L., Liu Y.H., Peng X.X.: Cloning, prokaryotic expression of 
rice L-galactono-1,4-lactone dehydrogenase gene and preparation of anti-GLDH antibodies. - J. Hunan Agric. Univ. 36: 381-384, 2010.

Yu L., Liu Y.H., Lu L. et al.: Ascorbic acid deficiency leads to increased grain chalkiness in transgenic rice for suppressed of
L-GalLDH. - J. Plant Physiol. 211: 13-26, 2017.

Zhang J., Li B., Yang Y. et al.: A novel allele of L-galactono-1,4lactone dehydrogenase is associated with enhanced drought tolerance through affecting stomatal aperture in common wheat. - Sci. Rep. 6: 30177, 2016. 